\title{
A Hybrid Importance Sampling Algorithm for Estimating VaR under the Jump Diffusion Model
}

\author{
Tian-Shyr Dai ${ }^{1}$; Li-Min Liu ${ }^{2}$ \\ ${ }^{1}$ Department of Information and Financial Management, Institute of Information Management and Institute of Finance, National \\ Chiao-Tung University, Taiwan ,China; ${ }^{2}$ Department of Applied Mathematics, Chung Yuan Christian University, Taiwan, \\ China. \\ Email: d88006@csie.ntu.edu.tw
}

Received July $20^{\text {th }}, 2009$; revised August $12^{\text {th }}, 2009$; accepted August $14^{\text {th }}, 2009$.

\begin{abstract}
Value at Risk (VaR) is an important tool for estimating the risk of a financial portfolio under significant loss. Although Monte Carlo simulation is a powerful tool for estimating VaR, it is quite inefficient since the event of significant loss is usually rare. Previous studies suggest that the performance of the Monte Carlo simulation can be improved by importance sampling if the market returns follow the normality or the distributions. The first contribution of our paper is to extend the importance sampling method for dealing with jump-diffusion market returns, which can more precisely model the phenomenon of high peaks, heavy tails, and jumps of market returns mentioned in numerous empirical study papers. This paper also points out that for portfolios of which the huge loss is triggered by significantly distinct events, naively applying importance sampling method can result in poor performance. The second contribution of our paper is to develop the hybrid importance sampling method for the aforementioned problem. Our method decomposes a Monte Carlo simulation into sub simulations, and each sub simulation focuses only on one huge loss event. Thus the performance for each sub simulation is improved by importance sampling method, and overall performance is optimized by determining the allotment of samples to each sub simulation by Lagrange's multiplier. Numerical experiments are given to verify the superiority of our method.
\end{abstract}

Keywords: Hybrid Importance Sampling, VaR, Straddle Options, Jump Diffusion Process

\section{Introduction}

Value at Risk (VaR) is an important tool for quantifying and managing portfolio risk. It provides a way of measuring the total risk to which the financial institution is exposed. VaR denotes a loss $\ell$ that will not be exceeded at certain confidence level $1-p$ over a time horizon from t to $t+\Delta t$. To be more specific,

$$
P\left(V_{t+\Delta t}-V_{t}<\ell\right)=p,
$$

where $V_{\tau}$ denotes portfolio value at time $\tau$. Typically, $p$ is close to zero. For convenience, we define $V_{t+\Delta t}-V_{t}$ and $\left(V_{t+\Delta t}-V_{t}\right) / V_{t}$ as the portfolio gain and the return over the time span $\Delta t$. Some academic papers focus on a relevant problem: computes the probability $p$ of a portfolio loss to exceed a given level $\ell$ [1], and our paper will focus on this problem.

VaR can not be evaluated by simple yet exact analytical formulas when the assumptions on the processes of the assets' values or the composition of financial portfolios are complex [2]. The asset in this paper is assumed to be stock for convenience. The Monte Carlo simulation is a flexible and powerful tool to estimate $\mathrm{VaR}$ since it is usually more easily to sample the stock prices from complex diffusion price processes than to estimate the distributions of the stock prices at a certain time point. We can repeatedly evaluate possible future values of a financial portfolio by sampling prices of stocks that compose the portfolio and the distribution of the portfolio gain can then be estimated. However, estimating VaR by the Monte Carlo simulation is very inefficient since the event that the portfolio loss exceeds $\ell$ is rare (note that $p$ is close to zero) and a large number of samples is thus required to obtain an accurate probability estimate of this rare event. By assuming the market returns follow normal distributions, Glasserman et al. develop an efficient variance reduction method based on importance sampling that can drastically reduce the number of samples required to achieve accurate probabilities estimates of rare events [3]. In their method, the stock prices are sampled from a new probability measure where the event of significant loss is more likely to happen than in the original one. This new probability measure is selected to 
"asymptotically minimize" the second moment of the estimator for estimating $P\left(V_{t+\Delta t}-V_{t}<\ell\right)$. (Details will be introduced in Section 2.)

Empirical studies claim that the stock returns observed from the real world markets show higher peaks and heavier tails than what is predicted by a normal distribution as illustrated in Figure 1 [4-6]. For estimating VaR, the heavy tail phenomenon must be taken into account since this phenomenon causes a significant loss of the stock price more likely to happen. To address this problem, Glasserman et al. extend their work by assuming that the stock returns follow t distributions [7]. Indeed, most financial papers address the aforementioned problem by assuming that the stock prices follow the jump-diffusion model [8], GARCH models [9], or the stochastic volatility model [10] instead of t distribution. The first contribution of this paper is to extend Glasserman et al. [7] to the jump diffusion model, which assumes that the stock returns and the jump sizes follow normal distributions and the arrival of jumps is modeled by a Poisson process. In this paper, the probability distributions of the stock returns, jump sizes, and the arrival of jumps are probably tilted to "asymptotically minimize" the second moment for estimating the probability of the huge loss event.

Glasserman's method performs poorly for portfolios of which huge loss is triggered by significantly distinct events. Take a portfolio, shorting straddle options (which will be introduced later), illustrated in Panel (a) of Figure 2 as an example. This portfolio suffers significant loss when the stock price increases or decreases drastically. Thus tilting the probability measure of the stock price to make one huge-loss event, says a significant decrease in the stock price, more likely to happen will make the other event (a significant increase in the stock price) much rarer. The numerical results in our paper show that

\section{probability density}

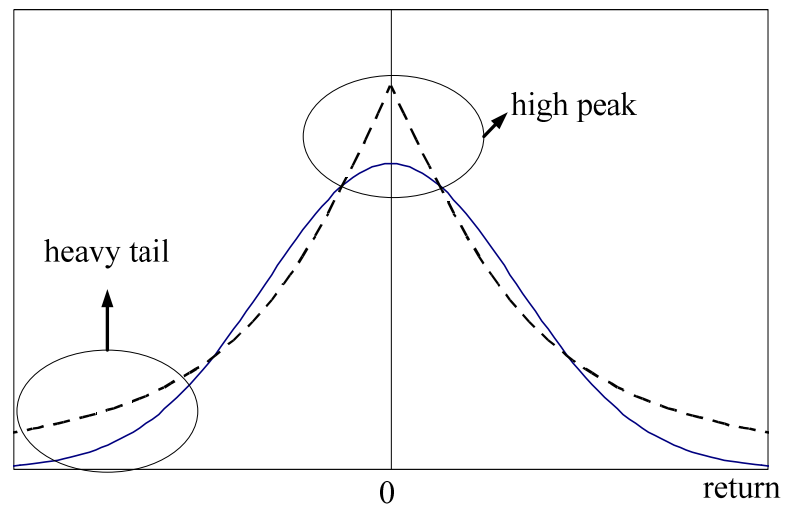

Figure 1. High peaks and heavy tails of stock returns

The solid line denotes the return modeled by a normal distribution and the dashed line denotes the return modeled by a t distribution, which is closer to the distribution of the real world market returns than the former distribution

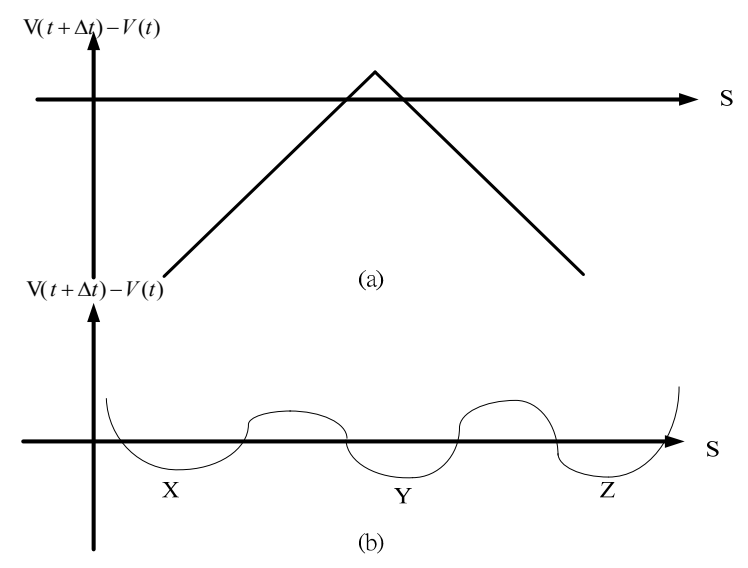

Figure 2. The relationship between the stock price and the portfolio gain

The $x$ - and $y$-axis denote the stock price and the portfolio gain, respectively. Panel (a) denotes the case of shorting straddle options near the option maturity date. Panel (b) denotes the case of three-minimum portfolio mentioned in [2]. $X, Y$, and $Z$ denotes there huge-loss events $\mathrm{f}$ this portfolio.

naively applying Glasserman's method deteriorates the performance. Glasserman et al. argue that the aforementioned problem can be solved by the delta-gamma approximation $[11,12]$ if the portfolio gain can be well approximated by a quadratic function of the stock price. But it is obvious that many portfolios, like the shorting straddle options and the three-minimum portfolio (see panel (b) of Figure 2) can not be well approximated by quadratic functions.

The second contribution of this paper is the hybrid importance sampling algorithm to solve the aforementioned problem. The hybrid importance sampling algorithm is composed of sub simulations; each sub simulation focuses on one significant loss event. For example, our algorithm for estimating the probability of huge loss for shorting straddle options can be decomposed into two sub simulations. On focuses on the significant decrease in the stock price and the other focuses on the significant increase in the stock price. The algorithm for the three-minimum portfolio can be decomposed into three sub simulations. These three sub simulations focus on huge-loss events X, Y, and Z, respectively. Each sub simulation tilts its probability measure of the stock price to "asymptotically minimize" the second moment for estimating the probability of the huge-loss event focused by that sub simulation. Finally, the computational resource allocated to each sub simulation is determined by Lagrange's multiplier to asymptotically minimize the second moment for estimating the overall huge loss probability.

Generally speaking, cross-discipline research, like bioinformatics and financial engineering, become more prevailing and important for both academics and practitioners. This paper merges the simulation technique from 
applied mathematics and algorithm design and performance comparisons knowhow from computer science discipline to develop efficient numerical programs to solve finance problem. It plays a great platform to interchange the ideas, the challenges, and the techniques among the computer scientists, mathematicians, and financial experts.

The paper is organized as follows. The assumptions of the Merton's jump diffusion model, the Glasserman's importance sampling method, and the definitions of straddle options are introduced in Section 2. In Section 3, we will use shorting straddle options as an example to demonstrate how the probabilities of the jump diffusion process are tilted for each sub simulation and how the number of samples is allocated to each sub simulation to optimize the overall performance. Numerical results in Section 4 verify the superiority of our method. Section 5 concludes the paper.

\section{Preliminaries}

\subsection{The Stock Price Process}

Define $S t$ as the stock price at year $t$. Under the Merton's jump diffusion model, the stock price process can be expressed as

$$
\frac{d S_{t}}{S_{t-}}=\mu d t+\sigma d W_{t}+\left(e^{X}-1\right) d N_{t}
$$

where $W_{t}$ is the standard Wiener process, $\mu$ is the average stock return per annum, $\sigma$ is the annual volatility, $X$ is a normal random variable that models the jump size, and $N_{t}$ denotes the Poisson process. We further assume that $X \sim N\left(\eta, \delta^{2}\right)$ and $P\left(d N_{t}=1\right)=\lambda d t$. Define the stock return over the time horizon $\Delta t$ as follows:

$$
r_{t} \equiv \frac{S_{t+\Delta t}-S_{t}}{S_{t}} \approx \mu \Delta t+\sigma \sqrt{\Delta t} Z+\sum_{i=1}^{N_{\Delta t}} Z_{i},
$$

where $Z \sim N(0,1), \quad N_{\Delta t}$ denotes the number of jumps between time $t$ and $t+\Delta t, Z_{i} \sim N\left(\eta, \delta^{2}\right)$. Note that the aforementioned model degenerates into the Black- Scholes lognormal diffusion process [13] when $\lambda=0$ (i.e. $N_{\Delta t}=0$ in Equation (2)).

\subsection{Glasserman's Importance Sampling Method}

This subsection sketches Glasserman's importance sampling method [3] by assuming that the stock price process follows the log-normal diffusion process. Consider a portfolio which is composed of a stock. Let $A$ denotes the event that the portfolio gain is less than $\ell$ :

$$
\begin{aligned}
A & \equiv\{S(t+\Delta t)-S(t)-\ell<0\} \\
& =\left\{\frac{\mathrm{S}(t+\Delta t)-\mathrm{S}(t)}{S(t)}-\frac{\ell}{S(t)}<0\right\} \\
& =\left\{r_{t}-\frac{\ell}{S(t)}<0\right\} \\
& =\left\{-r_{t}-r_{p}>0\right\} \\
& =\left\{\mathrm{Z}: \mathrm{f}(\mathrm{Z}) \equiv-r_{t}-r_{p}=-\mu \Delta t-\sigma \sqrt{\Delta t} Z-r_{p}>0\right\}
\end{aligned}
$$

where we substitute Equation (2) into Equation (3) and (5), and $r_{p} \equiv \frac{-\ell}{S_{t}}$ into Equation (4).

To minimize the second moment for estimating the probability of event $A$, Glasserman samples $Z$ from a new probability measure $\wp_{\theta}$ instead of the original probability measure $\wp \quad$ (where $Z \sim N(0,1))$. The likelihood ratio for these two probabilities measures is

$$
\frac{d \wp_{\theta}}{d \wp}=\exp \{\theta f(Z)-\Psi(\theta)\}
$$

where $\Psi(\theta) \equiv \log E[\exp (\theta f(Z))]$. Define $E_{\wp_{\theta}}$ as the expected value measured under $\wp_{\theta}$ and

$$
A_{\theta} \equiv\left\{Z_{\theta}: f\left(Z_{\theta}\right)=-\mu \Delta t-\sigma \sqrt{\Delta t} Z_{\theta}-r_{p}>0\right\},
$$

where $Z_{\theta} \sim N(\theta \sigma \sqrt{\Delta t}, 1)$. Then we have

$$
p=E\left(1_{A}\right)=E_{\wp_{\theta}}\left[1_{A_{\theta}} \exp \left(-\theta f\left(Z_{\theta}\right)+\Psi(\theta)\right)\right] . d * 9
$$

The second moment of the estimator is then

Second moment

$$
=E_{\wp_{\theta}}\left[1_{A_{\theta}} \exp \left(-2 \theta f\left(Z_{\theta}\right)+2 \Psi(\theta)\right)\right] \leq \exp (2 \Psi(\theta)) \text {. }
$$

To asymptotically optimize the performance of the Monte Carlo simulation, a proper $\theta$ is selected to minimize exp $(2 \Psi(\theta))$ by the following equation:

$$
\Psi^{\prime}(\theta)=0
$$

$\wp_{\theta}$ is then determined by substituting $\theta$ (obtained from Equation (8)) into Equation (6).

\subsection{Straddle Options}

Stock options are derivative securities that give their buyer the right, but not the obligation, to buy or sell the underlying stocks for a contractual price called the exercise price $K$ at maturity. Assume that the options mature at time $t+\Delta t$ then the payoffs of a call option and a put option at maturity are $\max \left(S_{t+\Delta t}-K, 0\right)$ and $\max$ $\left(K-S_{t+\Delta t}, 0\right)$, respectively. Shorting straddle options denotes a portfolio that shorts $D_{1}$ units call options and 
$D_{2}$ units put options with the same strike price. To be more specific, the portfolio gain at maturity is $-D_{1} \max \left(S_{t+\Delta t}-K, 0\right)-D_{2} \max \left(K-S_{t+\Delta t}, 0\right)$. The portfolio gain is interpreted in terms of stock return $r_{t}$ defined Equation (2) in Figure 3. Note that

$$
r_{0}^{*} \equiv \frac{K-S_{t}}{S_{t}}
$$

The portfolio gain can be expressed as follows:

$$
\text { Portfolio Gain }=\left\{\begin{array}{ll}
r_{0}^{*} S_{t} \delta_{2}+\left(r_{t}-r_{0}^{*}\right) S_{t} \delta_{1}, & \text { if } r_{\mathrm{t}} \geq r_{0}^{*}, \\
r_{t} S_{t} \delta_{2}, & \text { if } r_{\mathrm{t}}<r_{0}^{*},
\end{array},(10)\right.
$$

where $\delta_{1}=-D_{1}$ and $\delta_{2}=D_{2}$. The portfolio gain is less than $\ell$ if the stock return $r_{t}$ is larger than $r_{1}^{*}$ or lower than $r_{2}^{*}$, where

$$
r_{1}^{*}=\frac{\ell-r_{0}^{*} S_{t} \delta_{2}+r_{0}^{*} S_{t} \delta_{1}}{S_{t} \delta_{1}} \text { and } r_{2}^{*}=\frac{\ell}{S_{t} \delta_{2}} .
$$

\section{Contributions}

We will use shorting straddle options as an example to illustrate the major contributions of this paper. First, the huge loss events of this portfolio are identified. The Monte Carlo simulation is then decomposed into two sub simulations; each focuses on one huge loss event. Next, the probability distribution for each sub simulation is tilted to asymptotically minimize the second moment of the estimator under the jump diffusion assumption. Finally, the allotment of samples for each sub simulation is determined by Lagrange's multiplier to optimize the overall performance.

\subsection{Identify the Huge Loss Events}

In Figure 3, the portfolio gain of shorting straddle options is less than $\ell$ when the stock return $r_{t}$ exceeds threshold $r_{1}^{*}$ or is below $r_{2}^{*}$. For convenience, events $A_{1}$ and $A_{2}$ are used to denote the events $\left\{r_{t}>r_{1}^{*}\right\}$ and $\left\{r_{t}>r_{2}^{*}\right\}$, respectively, as follows:

$$
\begin{gathered}
A_{1} \equiv\left\{r_{t}: f_{1}\left(r_{t}\right) \equiv-r_{0}^{*} \delta_{2}-\left(r_{t}-r_{0}^{*}\right) \delta_{1}-r^{*}>0\right\}, \\
A_{2} \equiv\left\{r_{t}: f_{2}\left(r_{t}\right) \equiv-r_{t} \delta_{2}-r^{*}>0\right\},
\end{gathered}
$$

where $r^{*}=-\ell / S_{t}$ and formula $f_{1}\left(r_{t}\right)$ and $f_{2}\left(r_{t}\right)$ are derived from Equation (10). Since $A_{1}$ and $A_{2}$ are mutually exclusive, the probability that the portfolio gain is less than $\ell$ is

$$
p=E\left[1_{A_{1} \cup A_{2}}\right]=E\left[1_{A_{1}}\right]+E\left[1_{A_{2}}\right] .
$$

The Monte Carlo simulation for estimating $p$ can be decomposed into two sub simulations; one focuses on event $A_{1}$, and the other one focuses on event $A_{2}$.

\subsection{Importance Sampling under the Jump Diffusion Assumption}

Next, we will describe how to efficiently estimate $E\left[1_{A_{1}}\right]$ and $E\left[1_{A_{2}}\right]$ by importance sampling. Assume that the sub simulations for estimating $E\left[1_{A_{1}}\right]$ and $E\left[1_{A_{2}}\right]$ tilt their probabilities from $\wp$ to $\wp_{\theta_{1}}$ and $\wp_{\theta_{2}}$, respectively. Then $\theta_{1}$ and $\theta_{2}$ are derived as follows: Define $\Psi_{1}\left(\theta_{1}\right) \equiv \log E\left[\exp \left(\theta_{1} f_{1}\left(r_{t}\right)\right)\right]$ and $\Psi_{2}\left(\theta_{2}\right) \equiv \log E\left[\exp \left(\theta_{2} f_{2}\left(r_{t}\right)\right)\right]$. $E\left[\exp \left(\theta_{1} f_{1}\left(r_{t}\right)\right)\right]$ can be calculated as follows:

$$
\begin{array}{r}
E\left[\exp \left(\theta_{1} f_{1}\left(r_{t}\right)\right)\right]=E\left[\exp \left(\theta_{1}\left(-r_{0}^{*} \delta_{2}-r_{t} \delta_{1}+r_{0}^{*} \delta_{1}-r^{*}\right)\right)\right] \\
=\exp \left(-\theta_{1} r_{0}^{*} \delta_{2}+\theta_{1} r_{0}^{*} \delta_{1}-\theta_{1} r^{*}\right) E\left(\exp \left(-\theta_{1} r_{t} \delta_{1}\right)\right) .
\end{array}
$$

Note that

$$
\begin{aligned}
& E\left(\exp \left(-\theta_{1} r_{t} \delta_{1}\right)\right)=E\left[\exp \left(-\theta_{1} \delta_{1}\left(\mu \Delta t+\sigma \sqrt{\Delta t} Z+\sum_{i=1}^{N_{\Delta t}} Z_{i}\right)\right)\right] \\
& =\exp \left(-\theta_{1} \delta_{1} \mu \Delta t+0.5 \sigma^{2} \Delta t \theta_{1}^{2} \delta_{1}^{2}\right) E\left[\exp \left(-\theta_{1} \delta_{1} \sum_{i=1}^{N \Delta t} Z_{i}\right)\right] \text {. } \\
& E\left[\exp \left(-\theta_{1} \delta_{1} \sum_{i=1}^{N_{\Delta t}} Z_{i}\right)\right]=\sum_{n=0}^{\infty} \frac{e^{-\lambda_{t}} \lambda_{t}^{n}}{n !} E\left[\exp \left(-\theta_{1} \delta_{1} \sum_{i=1}^{n} Z_{i}\right)\right] \\
& =\sum_{n=0}^{\infty} \frac{e^{-\lambda_{t}} \lambda_{t}^{n}}{n !} \exp \left(-n \theta_{1} \delta_{1} \eta+0.5 n \theta_{1}^{2} \delta_{1}^{2} \delta^{2}\right. \\
& =\sum_{n=0}^{\infty} \frac{e^{-\lambda_{t}}\left(\lambda_{t} \exp \left(-\theta_{1} \delta_{1} \eta+0.5 \theta_{1}^{2} \delta_{1}^{2} \delta^{2}\right)\right)^{n}}{n !} \\
& =\exp \left(\lambda_{\mathrm{t}} e^{-\theta_{1} \delta_{1} \eta+0.5 \theta_{1}^{2} \delta_{1}^{2} \delta^{2}}-\lambda_{t}\right),
\end{aligned}
$$

where $\lambda_{t} \equiv \lambda \Delta t$. Thus $\theta_{1}$ can be obtained by numerically solving the equation $\Psi_{1}^{\prime}\left(\theta_{1}\right)=0$ (see Equation (8)) as follows:

$$
\begin{aligned}
& \Psi_{1}^{\prime}\left(\theta_{1}\right)=-r_{0}^{*} \delta_{2}+r_{0}^{*} \delta_{1}-r^{*}-\delta_{1} \mu \Delta t+\sigma^{2} \Delta t \theta_{1} \delta_{1}^{2} \\
& +\lambda_{\Delta t}\left(-\delta_{1} \eta+\theta_{1} \delta_{1}^{2} \delta^{2}\right) \exp \left(-\theta_{1} \delta_{1} \eta+0.5 \theta_{1}^{2} \delta_{1}^{2} \delta^{2}\right)=0 .
\end{aligned}
$$

Similarly, it can be derived that

$$
\begin{array}{r}
E\left[\exp \left(\theta_{2} f_{2}\left(r_{t}\right)\right)\right]=\exp \left(-\theta_{2} \delta_{2} \mu \Delta t+0.5 \sigma^{2} \Delta t \theta_{2}^{2} \delta_{2}^{2}\right. \\
\left.-\theta_{2} r^{*}+\lambda_{t} e^{-\theta_{2} \eta \delta_{2}+0.5 \theta_{2}^{2} \delta^{2} \delta_{2}^{2}}-\lambda_{t}\right) .
\end{array}
$$

$\theta_{2}$ can also be solved numerically by the equation $\Psi_{2}^{\prime}\left(\theta_{2}\right)=0$ as follows: 


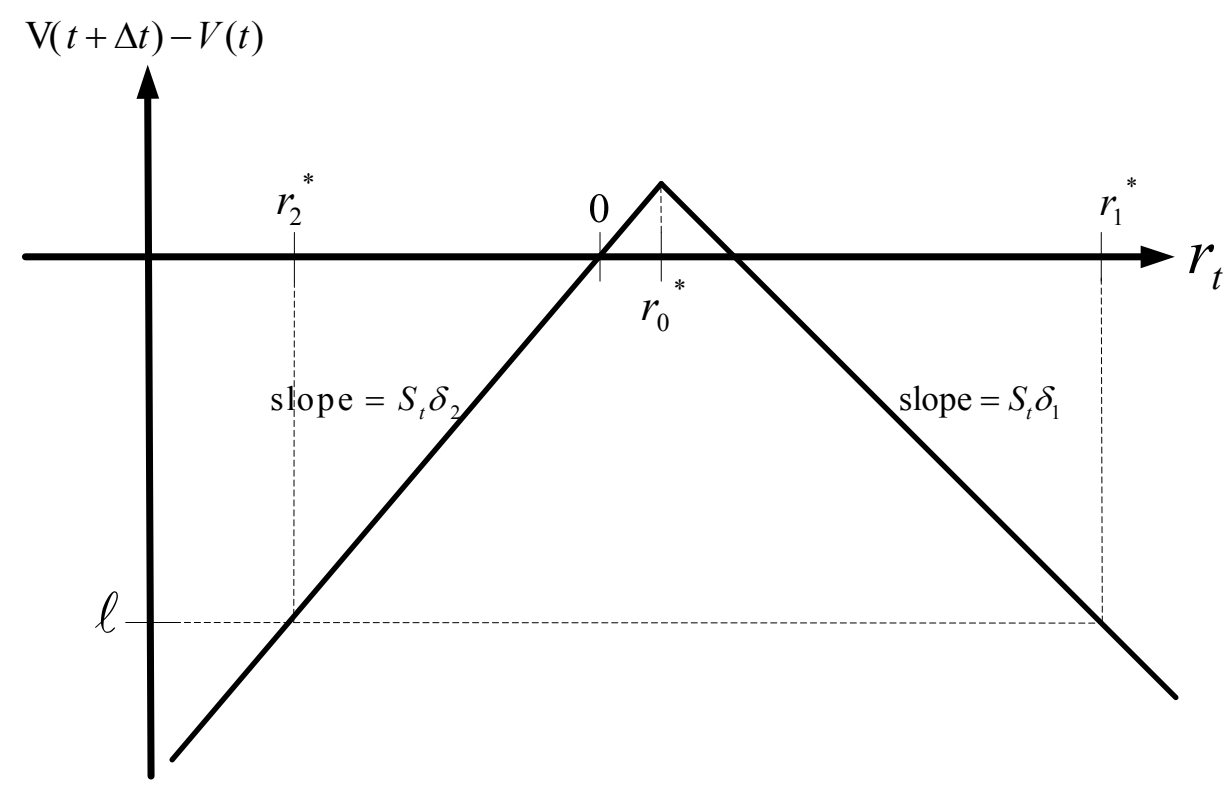

Figure 3. Shorting straddle options

The $x$ - and $y$-axis denote the stock return and the portfolio gain, respectively.

$$
\begin{aligned}
& \Psi_{2}^{\prime}\left(\theta_{2}\right)=-\delta_{2} \mu \Delta t+\sigma^{2} \Delta t \theta_{2} \delta_{2}^{2}-r^{*} \\
& +\lambda_{t}\left(-\eta \delta_{2}+\theta_{2} \delta^{2} \delta_{2}^{2}\right) e^{-\theta_{2} \eta \delta_{2}+0.5 \theta_{2}^{2} \delta^{2} \delta_{2}^{2}}=0 \text {. } \\
& d \wp_{\theta_{1}}=d \wp \exp \left(\theta_{1} f_{1}\left(r_{t}\right)-\Psi_{1}\left(\theta_{1}\right)\right) \\
& =\frac{1}{\sqrt{2 \pi}} e^{-Z^{2} / 2} \sum_{n=0}^{\infty}\left[\frac{e^{-\lambda_{t}} \lambda_{t}^{n}}{n !}\left(\frac{1}{\sqrt{2 \pi \delta^{2}}}\right)^{n} e^{-\frac{\sum_{k=1}^{n}\left(Z_{K}-\eta\right)^{2}}{2 \delta^{2}}}\right] \exp \left(\theta_{1} f_{1}\left(r_{t}\right)-\Psi_{1}\left(\theta_{1}\right)\right) \\
& =\frac{1}{\sqrt{2 \pi}} e^{-\frac{\left(Z+\theta_{1} \delta_{1} \sigma \sqrt{\Delta t}\right)^{2}}{2}} \sum_{n=0}^{\infty}\left[\frac{e^{-\lambda_{t} e^{-\theta_{1} \delta_{1} \eta+0.5 \theta_{1}^{2} \delta_{1}^{2} \delta^{2}}}\left(\lambda_{t} e^{-\theta_{1} \delta_{1} \eta+0.5 \theta_{1}^{2} \delta_{1}^{2} \delta^{2}}\right)^{n}}{n !}\left(\frac{1}{\sqrt{2 \pi \delta^{2}}}\right)^{n} e^{-\frac{\sum_{k=1}^{n}\left(Z_{K}-\left(\eta-\theta_{1} \delta_{1} \delta^{2}\right)\right)^{2}}{2 \delta^{2}}}\right] .
\end{aligned}
$$

That is, the first sub simulation tilts the probability from $\wp$ to $\wp_{\theta_{1}}$, where the distributions of random variables defined in Equation (2) are changed as follows:

$$
Z \sim N\left(-\theta_{1} \delta_{1} \sigma \sqrt{\Delta t}, 1\right)
$$

$N_{\Delta t} \sim$ Poisson $\left(\lambda_{t} e^{-\theta_{1} \delta_{1} \eta+0.5 \theta_{1}^{2} \delta_{1}^{2} \delta^{2}}\right)$ and $Z_{i} \sim N\left(\eta-\theta_{1} \delta_{1} \delta^{2}, \delta^{2}\right)$.

Note that

$$
\begin{aligned}
d \wp_{\theta_{2}} & =d \wp \exp \left(\theta_{2} f_{2}\left(r_{t}\right)-\Psi_{2}\left(\theta_{2}\right)\right) \\
= & \frac{1}{\sqrt{2 \pi}} e^{-\frac{\left(Z+\theta_{2} \delta_{2} \sigma \sqrt{\Delta t}\right)^{2}}{2}} \sum_{n=0}^{\infty}\left[\frac{e^{-\lambda_{t} e^{-\theta_{2} \delta_{2} \eta+0.5 \theta_{2}^{2} \delta_{2}^{2} \delta^{2}}}\left(\lambda_{t} e^{-\theta_{2} \delta_{2} \eta+0.5 \theta_{2}^{2} \delta_{2}^{2} \delta^{2}}\right)}{n !}\left(\frac{1}{\sqrt{2 \pi \delta^{2}}}\right)^{n} e^{\left.-\frac{\sum_{K=1}^{n}\left(Z_{K}-\left(\eta-\theta_{2} \delta_{2} \delta^{2}\right)\right)^{2}}{2 \delta^{2}}\right]}\right]
\end{aligned}
$$

$$
E\left[1_{A_{1}}\right]=E_{\wp_{\theta_{1}}}\left[1 A_{1} \exp \left(-\theta_{1} f_{1}\left(r_{t}\right)+\Psi_{1}\left(\theta_{1}\right)\right)\right] .
$$

Thus $E\left[1_{A_{1}}\right]$ is estimated by sampling the unbiased estimator $1_{A_{1}} \exp \left(-\theta_{1} f_{1}\left(r_{t}\right)+\Psi_{1}\left(\theta_{1}\right)\right)$ from $\wp_{\theta_{1}}$ in the first sub simulation. Similarly, the probability distribution $\wp_{\theta_{2}}$ used by the second sub simulation can be derived as 
Note also that

$$
E\left[1_{A_{2}}\right]=E_{\wp_{\theta_{2}}}\left[1_{A_{2}} \exp \left(-\theta_{2} f_{2}\left(r_{t}\right)+\Psi_{2}\left(\theta_{2}\right)\right)\right] .
$$

The second sub simulation estimates $E\left[1_{A_{2}}\right]$ by sampling the unbiased estimator $1_{A_{2}} \exp \left(-\theta_{2} f_{2}\left(r_{t}\right)+\Psi_{2}\left(\theta_{2}\right)\right)$ from $\wp_{\theta_{2}}$.

\subsection{Allocation of Computational Resources to Each Sub Simulation}

Finally, we try to minimize the upper bound of the second moment for estimating $p$ given a constraint on computational resources. The number of stock return samples serves as a proxy of computational resources. Assume that we can only sample $\mathrm{N}$ stock returns, and the numbers of stock returns sampled in the first and the second simulations are $n_{1}$ and $n_{2}$, respectively. By Equation (7), the upper bounds of the second moment of the estimator $1_{A_{1}} \exp \left(-\theta_{1} f_{1}\left(r_{t}\right)+\Psi_{1}\left(\theta_{1}\right)\right)$ and $1_{A_{2}} \exp \left(-\theta_{2} f_{2}\left(r_{t}\right)+\Psi_{2}\left(\theta_{2}\right)\right)$ under the probability measure $\wp_{\theta_{1}}$ and $\wp_{\theta_{2}}$ are $\exp \left(2 \Psi_{1}\left(\theta_{1}\right)\right)$ and $\exp \left(2 \Psi_{2}\left(\theta_{2}\right)\right)$, respectively. The second moment for estimating $p$ is then

$$
\frac{\exp \left(2 \Psi_{1}\left(\theta_{1}\right)\right)}{n_{2}}+\frac{2 \exp \left(\Psi_{2}\left(\theta_{2}\right)\right)}{n_{2}}
$$

To minimize Equation (14) under the constraint $n_{1}+n_{2}=\mathrm{N}, n_{1}$ and $n_{2}$ can be solved by Lagrange multiplier as follows:

$$
\begin{aligned}
& n_{1}=\frac{\sqrt{\exp \left(2 \Psi_{1}\left(\theta_{1}\right)\right)}}{\sqrt{\exp \left(2 \Psi_{1}\left(\theta_{1}\right)\right)}+\sqrt{\exp \left(2 \Psi_{2}\left(\theta_{2}\right)\right)}} \mathrm{N} \\
& n_{2}=\frac{\sqrt{\exp \left(2 \Psi_{2}\left(\theta_{2}\right)\right)}}{\sqrt{\exp \left(2 \Psi_{1}\left(\theta_{1}\right)\right)}+\sqrt{\exp \left(2 \Psi_{2}\left(\theta_{2}\right)\right)}} \mathrm{N}
\end{aligned}
$$

\section{Numerical Results}

Table 1 illustrates how the probability tilting mechanism proposed in this paper greatly improves the performance of the Monte Carlo simulation. Consider a portfolio which is composed of a stock. The probability that the portfolio loses more than $5 \%$ in 0.008 year is estimated with three different approaches: Original denotes the naive Monte Carlo simulation that samples the stock return from Equation (2). Lognormal denotes Glasserman et al. importance sampling method under the BlackScholes lognormal diffusion assumption (see subsection 2.2). Jump diffusion denotes the importance sampling method derived in Equation (13). We do 100 estimations
Table 1. Estimating the huge loss probability under different probability measures

\begin{tabular}{cccc}
\hline $\begin{array}{c}\text { Probability } \\
\text { Measure }\end{array}$ & Original & Lognormal & $\begin{array}{c}\text { Jump Dif- } \\
\text { fusion }\end{array}$ \\
\hline$\hat{p}$ & 0.0336 & 0.0339 & 0.0338 \\
$\hat{\operatorname{Var}}(\hat{p})$ & $2.49 \times 10^{-6}$ & $1.21 \times 10^{-6}$ & $3.69 \times 10^{-7}$ \\
\hline
\end{tabular}

The stock price is assumed to follow Merton's jump diffusion process: The stock average annual return $\mu$ is 0.05 , the annual volatility of the stock price $\sigma$ is 0.3 , the time span $\Delta t$ is 0.008 year, the jump frequency $\lambda$ is 6 , the average jump size $\eta$ is 0 , and the standard derivation of jump size $\delta$ is 0.03 . $\stackrel{\wedge}{p}$ and $\operatorname{Var}(\hat{p})$ denote the estimated probability and the variance, respectively.

for each Monte Carlo simulation method and each estimation samples 10000 stock returns. The probability for the portfolio to lose more than $5 \%$ is about $3.38 \%$. Obviously, the method proposed in this paper reduces the variance at a ratio of $\frac{3.69 \times 10^{-7}}{2.49 \times 10^{-6}} \approx 1 / 7$, which is better than the Glasserman's method $\left(\frac{1.21 \times 10^{-6}}{249 \times 10^{-6}} \approx 1 / 2\right)$.

Now we proceed to verify the superiority of the hybrid importance sampling algorithm in Table 2 and 3, where the stock price processes are assumed to follow the lognormal diffusion process and the Merton's jump diffusion process, respectively. The first column in these two tables denotes the probability measure of the stock return sampled by each Monte Carlo simulation method, where $\wp$ denotes the original probability measure defined in Equation (2), $\wp_{\theta_{1}}$ denotes the probability measure defined in Equation (12), and $\wp_{\theta_{2}}$ denotes the probability measure defined in Equation (13). Hybrid denotes the hybrid importance sampling algorithm that is composed of two sub simulations, which sample stock returns from $\wp_{\theta_{1}}$ and $\wp_{\theta_{2}}$, respectively. The numbers of samples allocated to these two sub simulations are determined in Equation (15) and (16), respectively. The second column $\hat{p}$ denotes the estimated probability for each Monte Carlo simulation, and the third column $\operatorname{Var}(\hat{p})$ denotes the variance of the estimated probability for each Monte Carlo simulation.

We first focus on Table 2. The event that the portfolio loss exceeds $\ell$ is about 0.0349 . This event can be decomposed into two mutually exclusive events $A_{1}$ and $A_{2}$ (see Equation (11)). The event $A_{1}$ (with probability $\left.P\left(A_{1}\right) \approx 0.0049\right)$ is less likely to happen than the event $A_{2}$ (with probability $P\left(A_{2}\right) \approx 0.0299$ ). Although tilting the probability measure of the stock return from $\wp$ to $\wp_{\theta_{1}}$ makes the Monte Carlo simulation estimate $P\left(A_{1}\right)$ more efficiently and accurately, it also damages the accuracy 
for estimating $P\left(A_{2}\right)$. It can be observed that this tilting produce inaccurate probability estimation $(0.0139)$ with large variance. Similar problem applies to the Monte Carlo simulation that tilts the probability measure to $\wp_{\theta_{2}}$; this Monte Carlo simulation is inadequate to estimate $P\left(A_{1}\right)$. But tilting the probability measure to $\wp_{\theta_{2}}$ is better than tilting the probability to $\wp_{\theta_{1}}$ since the event $A_{2}$ constitutes the bulk of the event that the portfolio loss exceeds $\ell$. Note that both important sampling methods mentioned above are less efficient than the primitive Monte Carlo simulation (that samples the stock return form $\wp)$. On the other hand, the hybrid importance sampling algorithm performs better than the primitive Monte Carlo simulation. It produces accurate probability estimation and reduces the variance at a ratio of $1 / 15\left(\approx \frac{2.28 \times 10^{-7}}{3.55 \times 10^{-6}}\right)$. In other words, the sample size of the primitive Monte Carlo simulation should be 15 times the sample size of the hybrid importance sampling algorithm to make the former method achieve the same accuracy level as the latter method.

In Table 3, the probability that the portfolio loss exceeds $\ell$ is larger (about 0.0403) since the jumps in stock price make the huge loss events $A_{1}$ and $A_{2}$ more likely to happen. Naively tilting the probability measure of the

Table 2. Compare monte carlo simulations under the lognormal stock price model

\begin{tabular}{ccccc}
\hline $\begin{array}{c}\text { Prob- } \\
\text { ability } \\
\begin{array}{c}\text { Meas- } \\
\text { ure }\end{array}\end{array}$ & $\wp$ & $\wp \theta_{1}$ & $\wp \theta_{2}$ & Hybrid \\
\hline$\hat{p}$ & 0.0349 & 0.0139 & 0.0395 & 0.0349 \\
$\hat{\wedge}$ & $3.55 \times 10^{-6}$ & $4.14 \times 10^{-3}$ & $2.52 \times 10^{-3}$ & $2.28 \times 10^{-7}$ \\
$\operatorname{Var}(\hat{p})$ & & & & \\
\hline
\end{tabular}

Consider a short straddle option that contains a short position in 1 unit call option $\left(\delta_{1}=-1\right)$ and 1 unit put option $\left(\delta_{2}=-1\right)$. The probability for the portfolio to lose more than $5 \%$ of the stock price in 0.008 year is estimated in column 2 (i.e., $r^{*}=0.05$ and $\ell \equiv-0.05 S_{t}$ ). The stock average annual return $\mu$ is 0.05 , the annual volatility of the stock price $\sigma$ is 0.3 , and $r_{0}^{*}$ defined in Equation (9) is 0.01 . Note that the jump frequency $\lambda$ is 0 in this case.

Table 3. Compare monte carlo simulations under the merton's jump diffusion stock price model

\begin{tabular}{ccccc}
\hline $\begin{array}{c}\text { Prob- } \\
\text { ability } \\
\begin{array}{c}\text { Meas- } \\
\text { ure }\end{array}\end{array}$ & $\wp$ & $\wp \theta_{1}$ & $\wp \theta_{2}$ & Hybrid \\
\hline$\hat{p}$ & 0.0405 & 0.0363 & 0.0426 & 0.0403 \\
$\begin{array}{c}\wedge \\
\operatorname{Var}(\hat{p})\end{array}$ & $4.05 \times 10^{-6}$ & $1.97 \times 10^{-2} s$ & $6.21 \times 10^{-4}$ & $5.44 \times 10^{-7}$ \\
\hline
\end{tabular}

The numerical settings follow the settings listed in Table 2 except that the jump frequency $\lambda$ is 6 , the mean of jump size $\eta$ is 0 , and the standard derivation of jump size $\delta$ is 0.03 . stock return to $P_{\theta_{1}}$ (or $P_{\theta_{2}}$ ) also performs poorly in this case. Note that the hybrid importance sampling algorithm still outperforms the primitive Monte Carlo simulation by reducing the variance at a ratio of $1 / 7.5\left(\approx \frac{5.44 \times 10^{-7}}{4.05 \times 10^{-6}}\right)$.

\section{Conclusions}

The paper improves the performance for estimating VaR. We first extend Glasserman's importance sampling method to Merton's jump diffusion process. Then we suggest a novel Monte Carlo simulation, the hybrid importance sampling algorithm, which can efficiently estimate the VaR of complex financial portfolios. Numerical results given in this paper verify that our method greatly improve the performance.

\section{Acknowledgement}

We thank Shi-Gra Lin, and Ren-Her Wang for useful suggestions.

\section{REFERENCES}

[1] S. K. Lin, C. D. Fuh, and T. J. Ko, "A bootstrap method with importance resampling to evaluate value-at-risk," J. Financial Studies, Vol. 12, pp. 81-116, 2004.

[2] H. G. Fong and K. C. Lin, "A new analytical approach to value at risk," J. Portfolio Management, Vol. 25, pp. 88-97, 1999.

[3] P. Glasserman, P. Heidelberger, and P. Shahabuddin, Vaniance Reduction Technique for Estimating Value-atRisk, Management Sci., Vol. 46, pp. 1349-1364, 2000.

[4] E. Eberlein, U. Keller, and K. Prause, "New insights into smile, mispricing, and value-at-risk: The hyperbolic model, J. Business, Vol. 71, pp. 371-406, 1998.

[5] J. R. M. Hosking, G. Bonti, and D. Siegel, "Beyond the Lognormal,” Risk, Vol. 13, pp. 59-62, 2000.

[6] K. Koedijk, R. Huisman, and R. Pownall, "VaR-x: Fat tails in financial risk management," J. Risk 1, pp. 47-62, 1998.

[7] P. Glasserman, P. Heidelberger, and P. Shahabuddin, "Portfolio value-at-risk with heavy-tailed risk factors," Math. Finance, Vol. 12, pp. 239-269, 2002.

[8] R. C. Merton, "Option pricing when underlying stock returns are discontinuous,” J. Financial Econ., Vol. 3, pp. 125-144, 1976.

[9] J.-C. Duan, "The GARCH option pricing model," Math. Finance, Vol. 5, pp. 13-32, 1995.

[10] S. Heston, "A closed-form folution for options with stochastic volatility with applications to bond and currency options," Rev. Financial Studies, Vol. 6, pp. 327-343, 1993.

[11] P. Jorion, Value at risk, McGraw-Hill, New York, 1997.

[12] C. Rouvnez, Going Greek with VaR, Risk 10 (1997) 57-65.

[13] F. Black and M. Scholes, "The pricing of options and corporate liabilities," J. Political Econ., Vol. 81, pp. 637-659, 1973. 